\title{
EXPLORANDO LA DIVERSIDAD DIETÉTICA EN LA PREHISTORIA DEL DESIERTO DE ATACAMA: UN ACERCAMIENTO A LOS PATRONES REGIONALES*
}

\author{
EXPLORING DIETARY DIVERSITY IN THE PREHISTORIC ATACAMA: \\ AN APPROXIMATION OF REGIONAL PATTERNS
}

\author{
William J. Pestle ${ }^{1}$, Christina Torres-Rouff ${ }^{2,5}$, Mark Hubbe $e^{3,5}$, \\ Francisca Santana S. ${ }^{4}$, Gonzalo Pimentel ${ }^{5}$, Francisco Gallardo ${ }^{6,7}$ y Kelly J. Knudson ${ }^{8}$
}

\begin{abstract}
A lo largo de su prehistoria, el norte árido de Chile fue ocupado por una gran diversidad de culturas dispersas por todos los pisos ecológicos de la región. Sin embargo, desde el Arcaico Tardío se encuentran evidencias de intercambio de recursos y elementos culturales entre estas zonas, práctica que se intensificó durante los Periodos Medio e Intermedio Tardío. En este trabajo se evaluó la composición de la dieta de las poblaciones locales, por medio del análisis de isótopos estables de carbono y nitrógeno. Los resultados muestran gran consumo de proteínas marinas en las poblaciones costeras y de regiones cercanas, junto con un posible aumento en el consumo de maíz a lo largo del tiempo. También se demuestra una considerable variedad en la dieta de los individuos de cada grupo, apoyando la idea de un constante flujo de personas y alimentos entre la costa y el altiplano durante la prehistoria de la región.

Palabras claves: desierto de Atacama, paleodieta, análisis de isótopos estables de carbono y nitrógeno.
\end{abstract}

During its prehistory, Chile's desert north was occupied by numerous cultures scattered across the various ecological niches of the region. However, from the Late Archaic forward there is archaeological evidence for the sharing of resources and cultural elements across groups, a practice that intensified during the Middle and Late Intermediate Periods. We explored the dietary composition of local populations through stable carbon and nitrogen isotope analyses. Our results show a high consumption of marine protein along the coast and nearby regions as well as a possible increase in maize consumption over time. Interestingly, these results show that there was substantial dietary variety internal to these groups. This supports the idea of a regular flow of people and food between the coast and the highlands throughout the prehistoric period.

Key words: Atacama Desert, paleodiet, carbon and nitrogen stable isotope analyses.

El norte de Chile (II Región) se caracteriza por sus extremos climáticos y ecológicos, siendo especialmente marcado por la presencia del desierto hiperárido de Atacama, el cual se encuentra limitado al oeste por las cordilleras de la costa y la franja costera del océano Pacífico, y al este por la imponente presencia de los Andes. A pesar de estas grandes barreras naturales, grupos humanos han ocupado la región desde el Arcaico Temprano, y evidencias de intercambio culturales y de recursos son comunes en el registro arqueológico desde por lo menos el Arcaico Tardío (p.ej., Núñez y Santoro 2011). Si bien investigaciones previas han planteado una importante influencia de los estados Andinos

* Artículo seleccionado del conjunto de ponencias presentadas en el III Congreso Latinoamericano de Arqueometría, realizado en Arica, Chile, el año 2011. Este manuscrito fue evaluado por investigadores externos y editado por Marcela Sepúlveda y Verónica Silva, en su calidad de editoras invitadas de la Revista.

1 Department of Anthropology, University of Miami, P.O. Box 248106, Coral Gables, FL, 33124-2005, U.S.A. w.pestle@ miami.edu

2 Department of Anthropology, University of California, Merced, 5200 North Lake Road, Merced, CA, 95343, U.S.A. ctorres-rouff@ucmerced.edu

3 Department of Anthropology, The Ohio State University, 4034 Smith Laboratory, 174 W. 18th Ave., Columbus, OH, 43210, U.S.A. hubbe.1@osu.edu

4 Research Laboratory for Archaeology and the History of Art, University of Oxford, Dyson Perrins Building, South Parks Rd, Oxford, OX1 3QY, UK. francisca.santanasagredo@st-hughs.ox.ac.uk

5 Instituto de Investigaciones Arqueológicas y Museo, Universidad Católica del Norte, Calle Gustavo Le Paige 380, San Pedro de Atacama, Chile. gpimentel@ucn.cl

6 Museo Chileno de Arte Precolombino, Bandera 361, Santiago, Chile.

7 Centro Interdisciplinario de Estudios Interculturales e Indígenas, Pontificia Universidad Católica de Chile, Campus San Joaquín, Avda. Vicuña Mackenna 4860, Macul, Santiago, Chile. fgallardo.ibanez@ gmail.com

8 Center for Bioarchaeological Research, School of Human Evolution and Social Change, Arizona State University, PO Box 872402, Tempe, AZ, 85287, U.S.A. kelly.knudson@asu.edu

Recibido: marzo 2013. Aceptado: septiembre 2013. 
(Tiwanaku e Inca) durante los periodos Medio e Intermedio Tardío en la organización del intercambio interregional (p.ej., Kolata 1993), trabajos recientes han extendido significativamente la evidencia de interacción cultural hacia el pasado (p.ej., Knudson et al. 2012; Pimentel et al. 2010), confirmando la idea de que el contacto entre regiones en los Andes Centro-Sur siempre tuvo un rol importante en el desarrollo cultural de las poblaciones locales, afectando además aspectos de su estilo de vida.

En el presente trabajo realizamos un primer intento de medir el impacto de la interacción y movilidad interregional en la dieta de los grupos prehistóricos de la región durante los Periodos Formativo (1.500 a.C.400 d.C), Medio (400-1.000 d.C.) e Intermedio Tardío (1.000-1.450 d.C.), mediante el análisis de isótopos estables de carbono y nitrógeno en restos humanos prehistóricos. Los individuos analizados representan poblaciones costeras, de los oasis y altiplano de la II Región de Chile, distribuidas a lo largo de los distintos pisos ecológicos de la zona. Por más que las barreras impuestas por las condiciones geológicas y climáticas de la región puedan ser vistas como factores determinantes en la dieta de los grupos locales, los resultados de este primer estudio sugieren un flujo significativo de recursos alimentarios a través de la región y sus respectivas barreras naturales.

\section{Contexto Arqueológico y Cronológico}

Los individuos incluidos aquí fueron analizados dentro del marco de investigación arqueológica de cada una de las localidades incluidas (Tabla 1). Detalles sobre los contextos originales de estos materiales pueden ser obtenidos en las publicaciones realizadas por cada proyecto (Knudson et al. 2012; Nado et al. 2012; Santana 2011; Torres-Rouff, Pestle y Gallardo 2012; Torres-Rouff, Pimentel y Ugarte 2012). Aquí se presentan los datos isotópicos obtenidos en cada uno de ellos, con el objetivo de ofrecer una primera perspectiva regional sobre la diversidad de la dieta asociada a la prehistoria regional. La muestra incluye grupos provenientes de la costa, pampa, oasis y altiplano andino, abarcando desde el periodo Formativo Temprano (1.500-500 a.C.) hasta el Intermedio Tardío (1.000-1.450 d.C.) (Figura 1 y

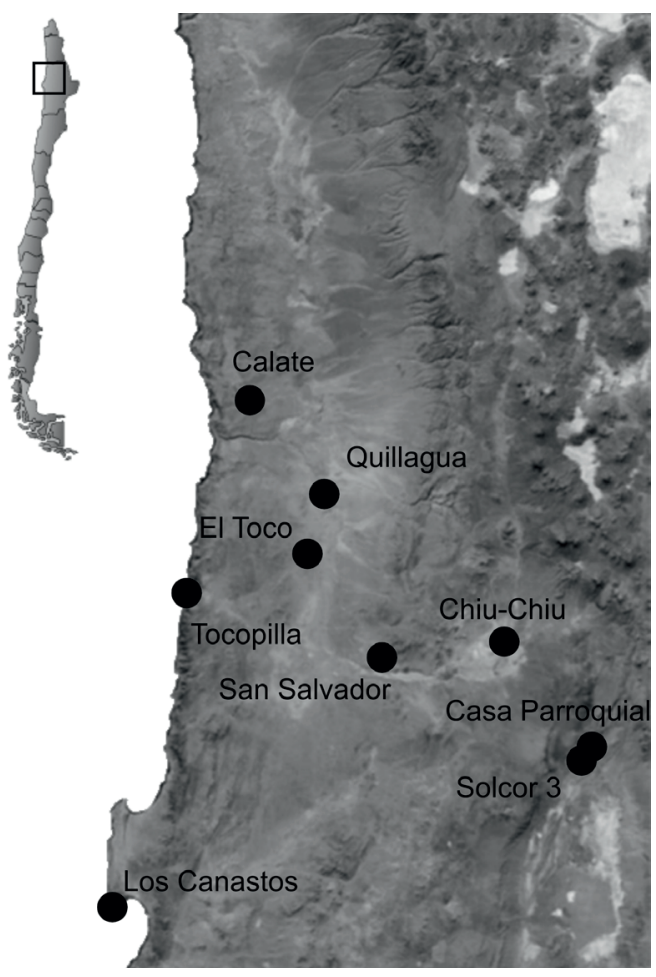

Figura 1. Ubicación geográfica de las muestras. Geographic location of the sample.

Tabla 1. Sitios incluidos en el estudio.

Study sample.

\begin{tabular}{lrll}
\hline \multicolumn{1}{c}{ Sitio } & N & \multicolumn{1}{c}{ Periodo } & \multicolumn{1}{c}{ Referencia } \\
\hline Los Canastos & 1 & Formativo Temprano (1.500-500 a.C.) & Isótopos inéditos \\
San Salvador & 9 & Formativo Medio (500 a.C.-100 d.C.) & Torres-Rouff; Pestle y Gallardo 2012 \\
Calate & 6 & Formativo Medio - Intermedio Tardío (500 a.C.-1.400 d.C.) & Pestle et al. 2012 \\
Chiu-Chiu & 3 & Formativo Tardío (100-400 d.C.) & Isótopos inéditos, Pollard 1970 \\
El Toco & 1 & Formativo Tardío (100-400 d.C.) & Cases et al. 2008; Knudson et al. 2012 \\
Casa Parroquial & 3 & Medio (400-1.000 d.C.) & Isótopos inéditos \\
Solcor 3 & 2 & Medio (400-1.000 d.C.) & Isótopos inéditos \\
Quillagua & 17 & Intermedio Tardío (1.000-1.450 d.C.) & Santana 2011 \\
Tocopilla & 2 & $?$ & Isótopos inéditos \\
\hline
\end{tabular}


Tabla 1). En términos de estilo de vida, los grupos incluidos en la muestra representan poblaciones pescadoras-cazadoras-recolectoras tempranas y grupos con distintos grados de sedentarización de periodos posteriores. La mayor parte de la muestra, sin embargo, está compuesta por individuos de grupos horticultores con mayor dependencia de maíz y plantas silvestres locales (p. ej., algarrobo), que mantuvieron una importante interacción interregional por medio de caravanas de llamas en rutas organizadas por Tiwanaku, durante el periodo Medio (400-1.000 d.C.), y por señoríos locales durante el periodo Intermedio Tardío (Tabla 1). Los análisis de los isótopos fueron realizados sobre muestras de huesos o dientes humanos. Aunque no sea representativa de todas las poblaciones estudiadas, la comparación de los resultados entre regiones ofrece una primera visión de los patrones de variación temporal y geográfica en la dieta de los grupos que habitaron el norte de Chile.

\section{Métodos}

Para reconstruir la dieta de cada individuo en la muestra, se determinaron los valores de $\delta^{13} \mathrm{C} \mathrm{y}$ de $\delta^{15} \mathrm{~N}$ a partir del colágeno de hueso, dentina y queratina de cabello y los valores de $\delta^{13} \mathrm{C}$ a partir de la apatita de hueso y esmalte dental, a través de la aplicación de espectrometría de masas. Los protocolos de extracción y el análisis de estos elementos variaron ligeramente entre los distintos laboratorios que participan en este trabajo, pero en general siguieron los procedimientos descritos en Longin (1971) y Ambrose (1990) para el colágeno y en Lee-Thorp (1989) y Koch et al. (1997) para la apatita de hueso y esmalte. Muestras con bajos niveles de preservación de colágeno fueron excluidas de los análisis (siguiendo los criterios de Ambrose 1990).

Los valores isotópicos obtenidos fueron corregidos para el fraccionamiento y posteriormente comparados con los valores de fuentes de alimentos vegetales y animales disponibles en la región (DeNiro y Hastorf 1985; Horn et al. 2009; Hückstädt et al. 2007; Minagawa and Wada 1984; Schoeninger y DeNiro 1984; Schoeninger et al. 1983; Tieszen y Chapman 1992; Torres-Rouff, Pestle y Gallardo 2012), que en general siguen patrones de variación isotópica conocidos. Debido a que plantas con diferentes sistemas fotosintéticos $\left(\mathrm{C}_{3}, \mathrm{C}_{4}\right.$ y $\left.\mathrm{CAM}\right)$ presentan señales isotópicas distintas para valores de $\delta^{13} \mathrm{C}$, los análisis de estos valores en muestras humanas nos permite discriminar la presencia de esos grupos de plantas en la dieta del individuo. En la región de estudio, el análisis de $\delta^{13} \mathrm{C}$ nos permite evaluar el consumo de maíz $\left(\mathrm{C}_{4}\right)$ en relación con la mayoría de las demás plantas de la región (p.ej., algarrobo), que presentan patrón fotosintético $\mathrm{C}_{3}$. Por su parte, el valor de $\delta^{15} \mathrm{~N}$ en tejidos animales varía de acuerdo con el nivel trófico que el animal ocupa en su ecosistema (DeNiro y Epstein, 1981; Schoeninger y DeNiro 1984; Schoeninger et al. 1983). Como la cadena trófica es más larga en el océano que entre animales terrestres, el valor de $\delta^{15} \mathrm{~N}$ en restos humanos presentará un valor más enriquecido en el caso de una dieta marina, lo que nos ayuda a inferir la importancia de los recursos costeros en la dieta de los individuos estudiados. Análisis realizados con animales de dieta controlada y restos arqueológicos (Ambrose y Norr 1993; Kellner y Schoeninger 2007) han demostrado que el valor de $\delta^{13} \mathrm{C}$ del colágeno es directamente influenciado por el consumo de proteínas en la dieta, mientras que valores de $\delta^{13} \mathrm{C}$ de apatita reflejan valores promedios derivados de todos los alimentos consumidos (proteínas, carbohidratos y lípidos), de tal manera que el análisis isotópico de colágeno y apatita permiten refinar la discusión sobre la composición básica de la dieta de cada individuo.

Para averiguar potenciales cambios cronológicos y espaciales en la dieta de los grupos del norte grande de Chile, los valores isotópicos fueron comparados y posteriormente correlacionados con la distancia de los sitios desde la costa y su periodo cronológico, para evaluar si estos factores explican la diversidad de dieta observada. Finalmente, todos los valores isotópicos humanos fueron comparados a una red alimentaria compuesta por 230 alimentos de los Andes centro-sur, incluyendo 132 muestras del norte árido de Chile, compilada desde los resultados de nuevos análisis nuestros (FONDECYT 1120376 y 1110702) y de datos publicados por otros autores (DeNiro y Hastorf 1985; Miller et al. 2010; Schoeninger y DeNiro 1984; Tieszen y Chapman 1992).

\section{Resultados}

Los valores de $\delta^{13} \mathrm{C}$ y $\delta^{15} \mathrm{~N}$ obtenidos para cada individuo pueden ser observados en la Tabla 2. En la Figura 2 presentamos la relación entre los valores de $\delta^{13} \mathrm{C}$ y $\delta^{15} \mathrm{~N}$ de colágeno, y en la Figura 3 presentamos la relación entre los valores de $\delta^{13} \mathrm{C}$ de apatita y $\delta^{15} \mathrm{~N}$ 
Tabla 2. Resultados de los valores de isótopos estables para cada individuo en la muestra. Stable isotope values for individuals included in the present study.

\begin{tabular}{|c|c|c|c|c|c|}
\hline Sitio & Periodo & Número & $\delta^{13} \mathrm{C}_{\mathrm{co}}$ & $\delta^{15} \mathrm{~N}_{\mathrm{co}}$ & $\delta^{13} \mathrm{C}_{\mathrm{ap}}$ \\
\hline Calate & Formativo Temprano & CH18-1-1 & $-16,72$ & 9,94 & $-11,93$ \\
\hline Calate & Medio & $\mathrm{CH} 2-5-1$ & $-15,44$ & 12,02 & $-11,63$ \\
\hline Calate & Formativo Tardío & CH3-7-1 & $-14,2$ & 22,61 & $-11,75$ \\
\hline Calate & Intermedio Tardío & CH3-8-1 & $-10,34$ & 28,02 & $-5,9$ \\
\hline Calate & Intermedio Tardío & $\mathrm{CH} 2-1-1$ & $-11,82$ & 26,33 & $-10,24$ \\
\hline Calate & Formativo Tardío & CH1-2-1 & $-11,83$ & 24,78 & $-9,82$ \\
\hline Casa Parroquial & Medio & CAP-0001 & $-13,74$ & 12,76 & \\
\hline Casa Parroquial & Medio & CAP-0002 & $-11,09$ & 11,92 & \\
\hline Casa Parroquial & Medio & CAP-0011 & $-11,21$ & 12,64 & \\
\hline Chiu-Chiu & Formativo Tardío & RAnL 273a-1\#1 & $-19,43$ & 11,28 & $-14,87$ \\
\hline Chiu-Chiu & Formativo Tardío & RAnL 273a-1\#2 & $-19,69$ & 11,16 & $-13,59$ \\
\hline Chiu-Chiu & Formativo Tardío & RAnL 273a-1\#3 & $-19,86$ & 11,31 & $-14,9$ \\
\hline El Toco & Formativo Tardío & $\mathrm{R} 1$ & $-12,7$ & 21,6 & $-8,95$ \\
\hline Los Canastos & Formativo Temprano & LC(6)-279-106 & $-12,77$ & 20,09 & $-9,35$ \\
\hline Quillagua & Intermedio Tardío & F11 diente & $-16,8$ & 14,5 & $-10,7$ \\
\hline Quillagua & Intermedio Tardío & I7 diente & $-16,3$ & 12,5 & -10 \\
\hline Quillagua & Intermedio Tardío & K16 Esq. 6 & $-13,9$ & 25,1 & $-9,8$ \\
\hline Quillagua & Intermedio Tardío & $\mathrm{L} 4$ & $-12,6$ & 22,4 & $-7,9$ \\
\hline Quillagua & Intermedio Tardío & H7 diente & $-12,8$ & 19,5 & $-7,7$ \\
\hline Quillagua & Intermedio Tardío & $\mathrm{C} 9$ & $-12,3$ & 23,4 & $-7,5$ \\
\hline Quillagua & Intermedio Tardío & F11 Esq. 15 & $-12,6$ & 16,7 & $-7,3$ \\
\hline Quillagua & Intermedio Tardío & E10 & $-12,4$ & 20,4 & $-7,1$ \\
\hline Quillagua & Intermedio Tardío & K8 & $-12,4$ & 17,3 & $-6,9$ \\
\hline Quillagua & Intermedio Tardío & F11 y F10 Esq. 14 & -12 & 16,4 & $-6,4$ \\
\hline Quillagua & Intermedio Tardío & K16 & $-12,1$ & 15,5 & $-6,3$ \\
\hline Quillagua & Intermedio Tardío & I16 diente & $-11,4$ & 18,4 & -6 \\
\hline Quillagua & Intermedio Tardío & G5 & $-11,1$ & 16,7 & $-5,8$ \\
\hline Quillagua & Intermedio Tardío & I11 & $-11,2$ & 14,4 & $-5,3$ \\
\hline Quillagua & Intermedio Tardío & H16 Esq. 8 & $-10,8$ & 15 & $-5,2$ \\
\hline Quillagua & Intermedio Tardío & $\mathrm{H} 5$ & -11 & 20,7 & $-5,1$ \\
\hline Quillagua & Intermedio Tardío & F8 & $-11,2$ & 20,1 & $-5,1$ \\
\hline San Salvador & Formativo Medio & 1.2 & $-17,43$ & 13,3 & $-13,26$ \\
\hline San Salvador & Formativo Medio & 9.1 & $-16,93$ & 15,37 & $-12,05$ \\
\hline San Salvador & Formativo Medio & 5.2 & $-16,17$ & 14,6 & $-11,25$ \\
\hline San Salvador & Formativo Medio & 1.1 & $-16,62$ & 11,24 & $-10,86$ \\
\hline San Salvador & Formativo Medio & 5.1 & $-15,47$ & 14,39 & $-10,62$ \\
\hline San Salvador & Formativo Medio & 3.1 & $-18,33$ & 12,94 & $-10,41$ \\
\hline San Salvador & Formativo Medio & 8.1 & -17 & 8,34 & $-9,91$ \\
\hline San Salvador & Formativo Medio & 10.1 & $-17,42$ & 10,57 & $-9,67$ \\
\hline San Salvador & Formativo Medio & 10.1 & $-15,49$ & 11,55 & $-9,36$ \\
\hline Solcor 3 & Medio & $1161 \mathrm{~A}$ & $-17,6$ & 10,1 & $-10,5$ \\
\hline Solcor 3 & Medio & 1558 & $-15,9$ & 11,4 & \\
\hline Tocopilla & $?$ & AlNT-57 & $-11,9$ & 23,36 & $-8,06$ \\
\hline Tocopilla & $?$ & CT-ju & $-11,57$ & 24,86 & $-8,91$ \\
\hline
\end{tabular}

de colágeno. Los resultados muestran valores altos para $\delta^{15} \mathrm{~N}$ y una alta diversidad entre regiones: más de $20 \%$ en $\delta^{15} \mathrm{~N}$ y casi $-10 \%$ en $\delta^{13} \mathrm{C}$ de colágeno y apatita. Sin embargo, también se observa una sorprendente cantidad de variación dentro de cada sitio. El rango de valores observados en $\delta^{15} \mathrm{~N}$ para cualquier valor de $\delta^{13} \mathrm{C}_{\text {ap }}$ sugiere un alto consumo de proteínas marinas, terrestres y de plantas $\mathrm{C} 3 / \mathrm{C} 4$ entre los grupos ${ }^{1}$. Además, es notable la diversidad de dietas entre individuos de un mismo sitio, como por ejemplo el caso de Quillagua.

El análisis de los valores de $\delta 13$ Cap y $\delta 15 \mathrm{~N}$ por periodo manifiesta tendencias diacrónicas interesantes (Figura 4). Tanto en los valores de carbono como en los de nitrógeno hay una correlación positiva significativa con el periodo cronológico 


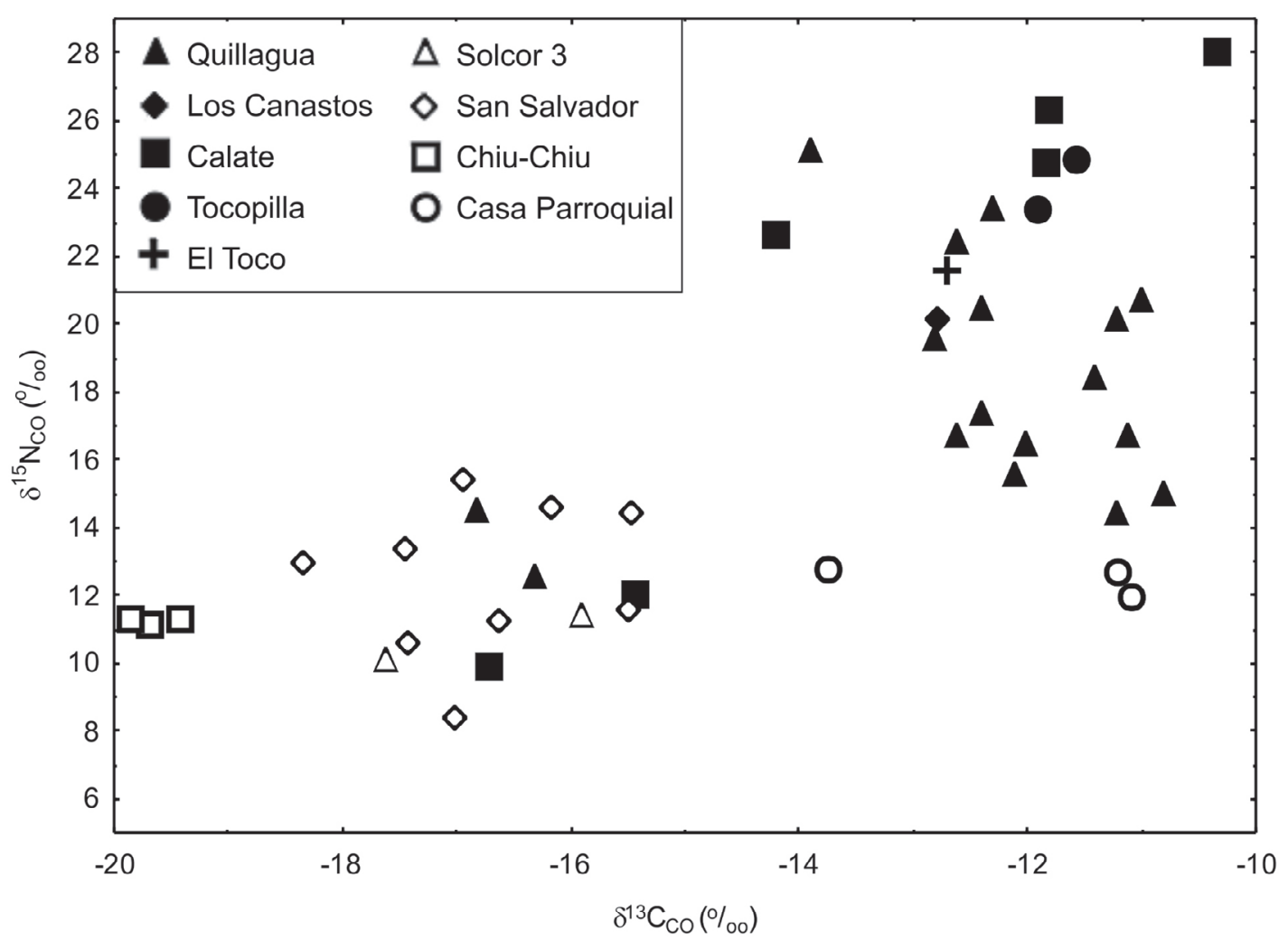

Figura 2. Gráfico con los valores de $\delta^{13} \mathrm{C}$ y $\delta^{15} \mathrm{~N}$ de colágeno de cada individuo. Graph of the $\delta^{13} C_{a p}$ and $\delta^{15} N_{c o}$ values for each individual.

$\left(\mathrm{r}=0,69 ; \mathrm{p}<0,001\right.$ para $\delta^{13} \mathrm{C}_{\mathrm{ap}} ; \mathrm{r}=0,41 ; \mathrm{p}=0,046$ para $\left.\delta^{15} \mathrm{~N}\right)$. Consecuentemente, los valores $\delta^{13} \mathrm{C}_{\mathrm{ap}}$ presentan un considerable enriquecimiento a lo largo del tiempo, lo cual refleja un aumento diacrónico en el consumo de maíz (C4). Para el nitrógeno, la correlación es menor, reflejando una disminución en la ingesta de recursos marinos a través del tiempo en la región. Sin embargo, dado que las muestras tardías son provenientes del interior, es probable que esto sea una tendencia exacerbada o generada por la ausencia de poblaciones costeras tardías en la muestra.

En lo que se refiere a la localidad geográfica de los grupos, no se observa una correlación significativa entre $\delta^{13} C_{a p}(r=-0,24 ; p=0,128)$, mostrando que no hay cambios en el consumo de plantas $\mathrm{C} 3$ y C4 entre el interior y la costa. Por otro lado, hay una correlación negativa significativa entre $\delta^{15} \mathrm{~N}$ y distancia de la costa $(r=-0,64 ; p<0,001)$, mostrando claramente que los grupos más lejanos de la costa tenían menor acceso a fuentes de proteínas marinas, como esperado.

\section{Discusión y Conclusiones}

A escala regional, los resultados de este estudio sugieren una enorme variedad y complejidad en la dieta de las poblaciones, claramente asociada a distintas estrategias de consumo de carbohidratos y proteínas. Los valores de $\delta^{13} \mathrm{C}$ y $\delta^{15} \mathrm{~N}$ muestran individuos con regímenes de consumo casi exclusivo de plantas C3 (algarrobo y otros frutos locales) y proteínas terrestres, como es el caso en Chiu-Chiu, hasta una dependencia casi completa de carbohidratos C4 (maíz) y proteínas marinas en algunos de los individuos de Calate.

Más interesante que esta variación regional, sin embargo, es el grado de diversidad de dietas encontrada entre individuos de un mismo grupo. En San Salvador y Quillagua hay un grado inmenso de variación en el consumo de proteínas y plantas (con un rango entre $7 \%$ y $13 \%$ o de $\delta^{15} \mathrm{~N}$ y de $-4 \%$ o a $-6 \%$ en $\delta^{13} \mathrm{C}_{\text {ap }}$, respectivamente). Es probable que una diversidad de tal magnitud no se deba en forma exclusiva al resultado de la circulación de productos 


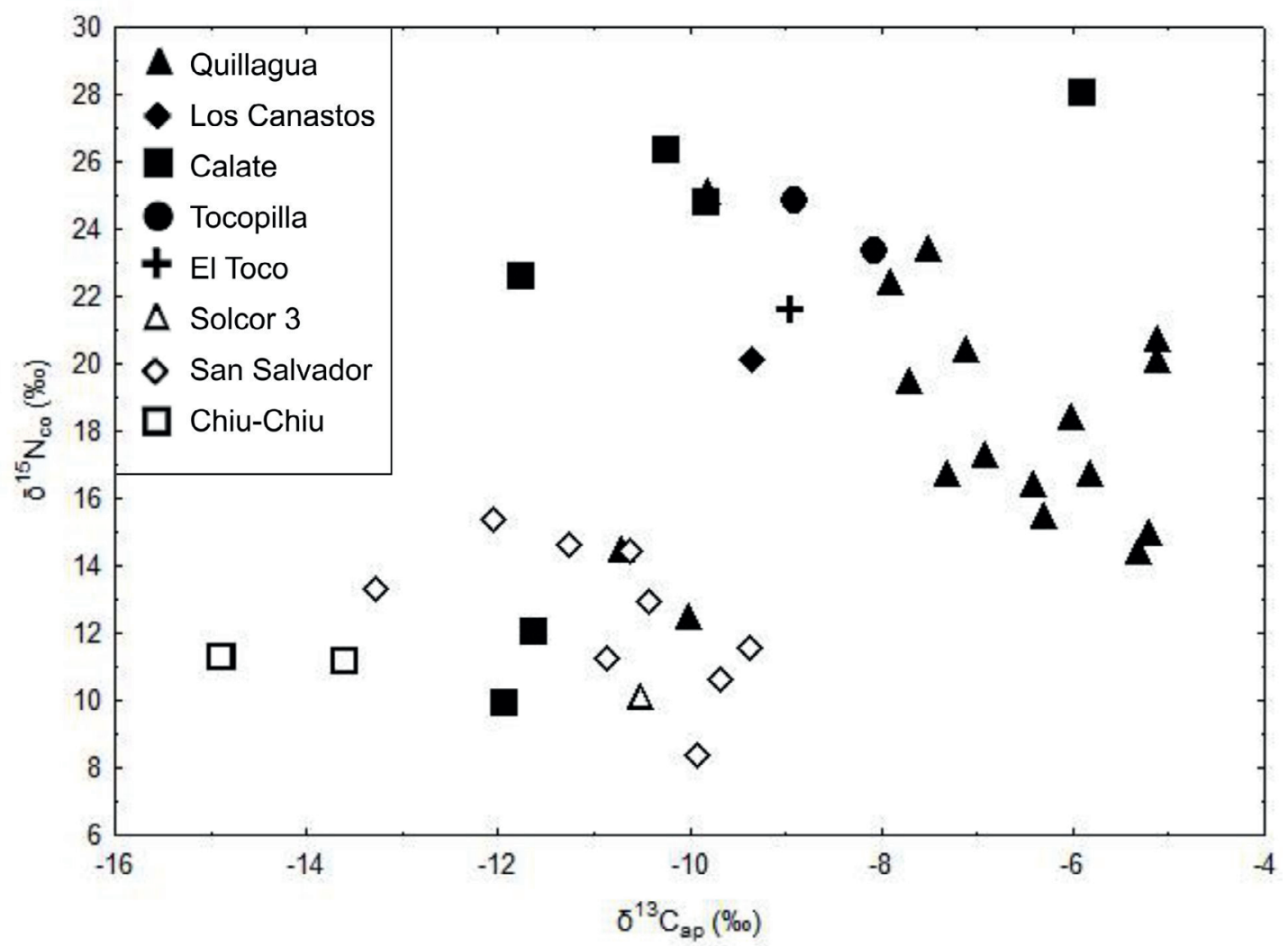

Figura 3. Gráfico con los valores de $\delta^{13} \mathrm{C}$ de apatita y $\delta^{15} \mathrm{~N}$ de colágeno de cada individuo. Graph of the $\delta^{13} C_{a p}$ and $\delta^{15} N_{c o}$ values for each individual.

alimentarios (dado que estos datos óseos reflejan el promedio de los últimos 10 años de la vida del individuo, reflejando más bien una consecuencia del movimiento de personas entre los distintos pisos ecológicos de la región o diferencias sociales en el acceso a determinados tipos de alimento. Esto es particularmente evidente en Quillagua, un sitio $70 \mathrm{~km}$ distante de la costa, donde los altos valores de nitrógeno sugieren un consumo significativo de proteínas marinas por los individuos. Santana (2011) ha descrito también, a partir de análisis de isótopos de oxígeno, la presencia de cuatro individuos foráneos con probable origen en el altiplano.

El movimiento bidireccional de individuos desde y hacia la costa se ve confirmado por los valores de los isótopos de los viajeros excavados en Calate (Pestle et al. 2012; Torres-Rouff, Pimentel y Ugarte 2012), que representan una muestra de individuos que fallecieron mientras viajaban por las rutas caravaneras que conectaban la costa con la pampa. Este hallazgo sugiere una mayor dinámica de movilidad poblacional en la región de lo que se había considerado en modelos anteriores (p.ej., Murra 1972), mismo para los periodos preincaicos, donde tradicionalmente no se ha considerado un contacto sistemático entre grupos altiplánicos y costeros. En otras palabras, nuestros datos corroboran estudios recientes (Pimentel et al. 2010; Santana 2011), que sugieren que la dinámica de intercambio interregional fue tan importante en la costa como lo fue en las tierras altas.

Finalmente, la movilidad estacional se ve reflejada en los análisis de isótopos de cabello de un individuo de El Toco (Knudson et al. 2012), quien muestra una periodicidad en los viajes y una estadía larga en cada una de las regiones visitadas, mucho más extensas de lo necesario para una expedición de comercio directo aunque no sea claramente estacional. Mientras que la motivación específica para una circulación de tan larga duración es desconocida, ella claramente apoya la idea de que la interacción costa-interior fue más frecuente y compleja de lo que se pensaba anteriormente. 


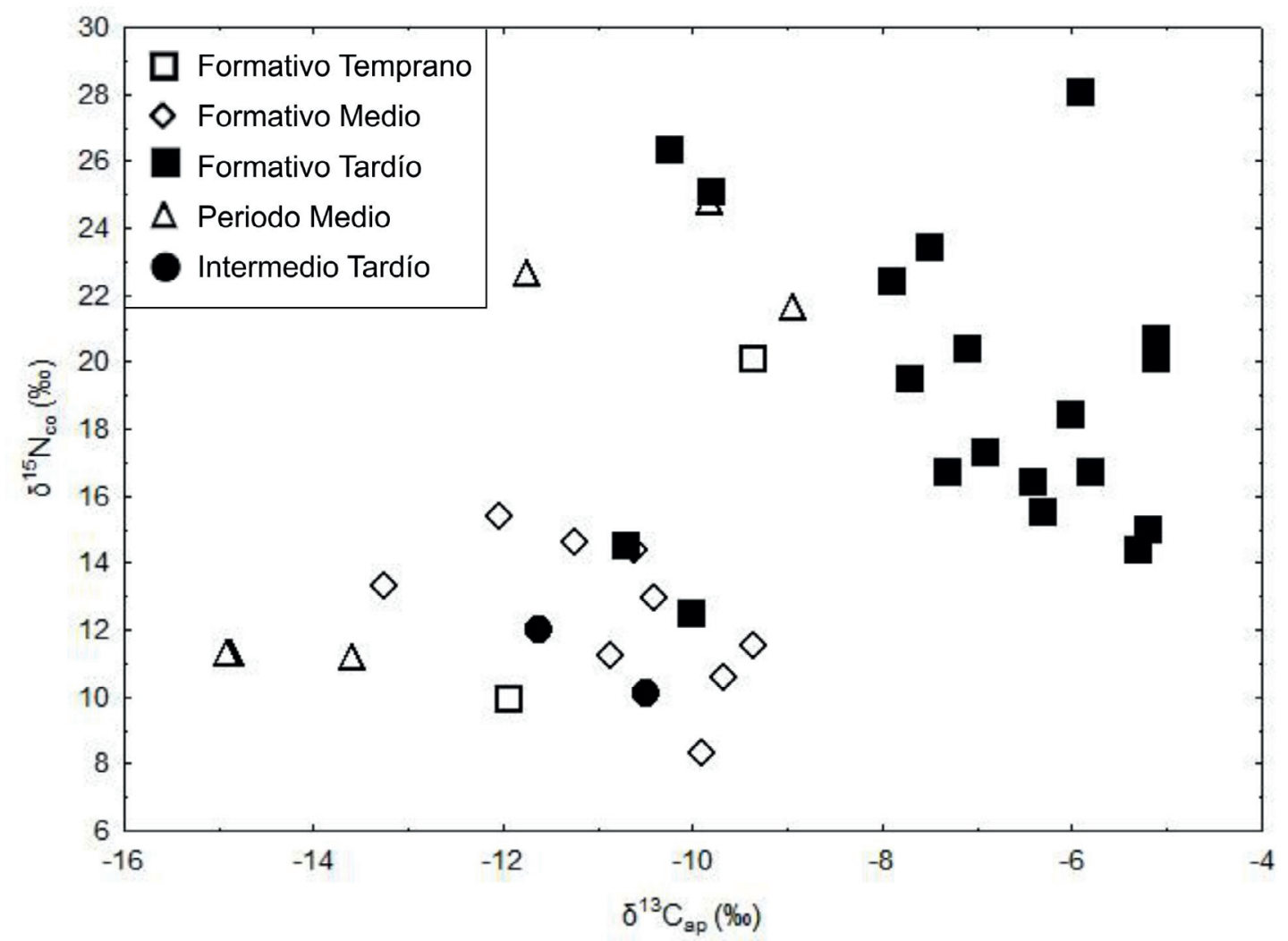

Figura 4. Gráfico con los valores de $\delta^{13} \mathrm{C}$ apatita y $\delta^{15} \mathrm{~N}$ de colágeno por periodo.

Graph of the $\delta^{13} C_{a p}$ and $\delta^{15} N_{c o}$ values by period.

En conclusión, si bien los resultados presentados aquí no son extensos, ellos muestran claramente la complejidad de los patrones de movimiento e interacción a lo largo de esta inhóspita región de Chile. Aun así, debe ser destacado que el presente trabajo está limitado por las distintas naturalezas de las preguntas realizadas en cada una de las regiones incluidas en nuestra comparación (p.ej., Quillagua fue investigado por uno de los autores (Santana 2011) con un enfoque en la etnicidad tardía, mientras que los datos de San Salvador resultan de un proyecto enfocado en la movilidad (Torres-Rouff, Pestle y Gallardo 2012). Esta realidad dificulta la construcción de un cuadro comparativo que considere las variaciones locales derivadas de aspectos ecológicos específicos, como por ejemplo tasas distintas de asimilación de nitrógenos por el efecto de fertilizantes o fruto de la aridez del clima. Sin embargo, los resultados presentados sirven para provocar una discusión regional y proponer hipótesis que pueden a partir de ese momento ser probadas en los contextos locales con el desarrollo de los estudios refinados que cada uno de nosotros está conduciendo en las distintas regiones comparadas aquí. Las contribuciones de dichos proyectos en los próximos años ciertamente enriquecerán el cuadro preliminar planteado en este estudio exploratorio.

Agradecimientos: Agradecemos el apoyo de NSF BCS-0721229 (CTR) y BCS-0721388 (KJK), FONDECYT 11070091 (MH), 1090762 (GP), 1120376 (CTR), y 1110702 (FG), FONDAP 15110006 (FG) y de nuestras instituciones. También reconocemos a las contribuciones de Blair Daverman, Francisca Fernández, Amy González, Laura King, Jimena Reséndiz y Mauricio Uribe (FONDECYT 1080458). Finalmente, agradecemos a los evaluadores del manuscrito por sus valiosas sugerencias y comentarios. 


\section{Referencias Citadas}

Ambrose, S.H. 1990. Preparation and characterization of bone and tooth collagen for isotopic analysis. Journal of Archaeological Science 17:431-451.

Ambrose, S.H. 1991. Effects of diet, climate and physiology on nitrogen isotope abundances in terrestrial foodwebs. Journal of Archaeological Science. 18:293-317.

Ambrose, S.H. y L. Norr 1993. Experimental evidence for the relationship of the carbon isotope ratios of whole diet and dietary protein to those of bone collagen and carbonate. En Prehistoric Human Bone: Archaeology at the Molecular Level, editado por J.B. Lambert y G. Grupe, pp. 1-37. Springer-Verlag, New York.

Cases, B., Ch. Rees, G. Pimentel, R. Labarca y D. Leiva 2008. Sugerencias desde un contexto funerario en un "espacio vacío" del Desierto de Atacama. Boletín del Museo Chileno de Arte Precolombino 13:51-70.

DeNiro, M.J. y S. Epstein 1981. Influence of diet on the distribution of nitrogen isotopes in Animals. Geochimica et Chosmochimica Acta 45:341-351.

DeNiro, M.J. y C. Hastorf 1985. Alteration of ${ }^{15} \mathrm{~N} /{ }^{14} \mathrm{~N}$ and ${ }^{13} \mathrm{C} /{ }^{12} \mathrm{C}$ ratios of plant matter during the initial stages of diagenesis: Studies using archaeological specimens from Peru. Geochimica et Chosmochimica Acta 49:97-115.

Horn, P., S. Holzl, S. Rummel, G. Aberg, S. Schiegel, D. Biermann, U. Struck y A. Rossman 2009. Humans and camelids in river oases of the Ica-Palpa-Nazca Region in Pre-Hispanic Times-Insights from H-C-N-O-S-Sr Isotope Signatures. En New Technologies for Archaeology, editado por M. Reindel y G.A. Wagner, pp. 173-192. Springer-Verlag, Berlin.

Hückstädt, L.A., C.P. Rojas y T. Antezana 2007. Stable isotope analysis reveals pelagic foraging by the Southern Sea Lion in Central Chile. Journal of Experimental Marine Biology and Ecology 347:123-133.

Kellner, C.M. y M.J. Schoeninger 2007. A Simple carbon isotope model for reconstructing prehistoric human diet. American Journal of Physical Anthropology 133:1112-1127.

Knudson, K.J., W.J. Pestle, C. Torres-Rouff y G. Pimentel 2012. Assessing the life history of an andean traveller through biogeochemistry: stable and radiogenic isotope analysis of archaeological human remains from Northern Chile. International Journal of Osteoarchaeology 22:435-451.

Koch, P.L., N. Tuross y M.L. Fogel 1997. The effects of sample treatment and diagenesis on the isotopic integrity of carbonate in biogenic hydroxyapatite. Journal of Archaeological Science 24:417-429.

Kolata, A. 1993. The Tiwanaku: Portrait of an Andean Civilization. Blackwell, Cambridge.

Lee-Thorp, J.A. 1989. Stable Carbon Isotopes in Deep Time: The Diets of Fossil Fauna and Hominids. Department of Archaeology, University of Cape Town, South Africa.

Longin, R. 1971. New method of collagen extraction for radiocarbon dating. Nature 230:241-242.

Miller, M.J., J.M. Capriles y C.A. Hastorf 2010. The fish of Lake Titicaca: Implications for archaeology and changing ecology through stable isotope analysis. Journal of Archaeological Science 37:317-327.

Minagawa, M. y E. Wada 1984. Stepwise enrichment of ${ }^{15} \mathrm{~N}$ along food chains: Further evidence and the relation between $\delta^{15} \mathrm{~N}$ and animal age. Geochimica et Cosmochimica Acta 48:1135-1140.

Murra, J.V. 1972. El "Control Vertical" de un máximo de pisos ecológicos en la economía de las sociedades andinas. En Visita de la Provincia de Leon de Huanuco en 1562, editado por John V. Murra, Vol. 2, pp. 429-476. Universidad Nacional Hermilio Valdizan, Huánuco, Peru.

Nado, K.L., S.J. Marsteller, L.M. King, B.M. Daverman, C. Torres-Rouff y K.J. Knudson 2012. Examining local social identities through patterns of biological and cultural variation in the Solcor Ayllu, San Pedro de Atacama. Chungara Revista de Antropología Chilena 44:239-255.

Núñez, L. y C.M. Santoro 2011. El tránsito arcaico-formativo en la circumpuna y valles occidentales del centro sur andino: hacia los cambios 'neolíticos'. Chungara Revista de Antropología Chilena 43:487-530.

Pestle, W.J., C. Torres-Rouff, G. Pimentel y K.J. Knudson 2012. Living and dying along the atacama's prehistoric routes: Analysis of human skeletal remains from Chile's Absolute Desert. Manuscrito en posesión del autor.

Pimentel, G., Ch. Rees, P. de Souza y L. Arancibia 2010. Viajeros costeros y caravaneros. Dos estrategias de movilidad transversal del Periodo Formativo (Desierto de Atacama, Chile). En En Ruta: Arqueología, Historia y Etnografía del Tráfico Surandino, editado por L. Núñez y A. Nielsen, pp. 43-81. Editorial Brujas, Córdoba.

Pollard, G. 1970. The Cultural Ecology of Ceramic-Stage Settlement in the Atacama Desert. University Microfilms, Inc., Ann Arbor.

Santana, F. 2011. Multiculturalidad en el Cementerio Oriente de Quillagua: Una Aproximación desde la Bioantropología Mediante Análisis Isotópicos de Dieta y Movilidad en el Curso Inferior del Río Loa, Periodo Intermedio Tardío. Tesis de grado, Universidad de Chile, Santiago.

Schoeninger, M.J. y M.J. DeNiro 1984. Nitrogen and carbon isotopic composition of bone collagen from marine and terrestrial animals. Geochimica et Cosmochimica Acta 48:625-639.

Schoeninger, M.J., M.J. DeNiro y H. Tauber 1983. Stable nitrogen isotope ratios of bone collagen reflect marine and terrestrial components of prehistoric human diet. Science 220:1381-1383.

Schwarcz, H. 1999. 15N enrichment in the Sahara: In search of a global relationship. Journal of Archaeological Science 26:629-636.

Sponheimer, M., T. Robinson, L. Ayliffe, B. Roeder, J. Hammer, B. Passey, A. West, T. Cerling, D. Dearing y J. Ehleringer 2003. Nitrogen isotopes in mammalian herbivores: Hair $\delta^{15} \mathrm{~N}$ values from a controlled feeding study. International Journal of Osteoarchaeology 13:80-87. 
Szpak, P., F.J. Longstaffe, J.-F. Millaire y C.D. White 2012. Stable isotope biogeochemistry of seabird guano fertilization: Results from growth chamber studies with maize (Zea mays). PLoS One 7:1-16.

Tieszen, L.L. y M. Chapman 1992. Carbon and nitrogen isotopic status of the major marine and terrestrial resources in the Atacama Desert of Northern Chile. Proceedings of the First World Congress on Mummy Studies, pp. 409-425. Museo Arqueológico y Etnográfico de Tenerife, Tenerife.
Torres-Rouff, C., W.J. Pestle y F. Gallardo 2012. Eating fish in the driest desert in the world: Osteological and biogeochemical analyses of human remains from the San Salvador Cemetery, North Chile. Latin American Antiquity 23:51-69.

Torres-Rouff, C., G. Pimentel y M. Ugarte 2012. ¿Quiénes viajaban? Investigando la muerte de viajeros post arcaicos en el Desierto de Atacama (ca. 800 AC-1536 DC). Estudios Atacameños: Arqueología y Antropología Surandina 43: 167-186.

\section{Nota}

1 Es posible que una pequeña parte de la elevación observada en los valores humanos de $\delta 15 \mathrm{~N}$ se deba al resultado del consumo de plantas fertilizadas con guano de aves marinas, la aridez, o el posible enriquecimiento de nitrógeno observado en animales como alpacas por efecto de su fisiología intestinal (Ambrose 1991; Schwarcz 1999; Szpak et al. 2012; Sponheimer et al 2003). 
